\title{
SAVED BY THE APPENDIX - A CASE OF SEROUS CYSTADENOCARCINOMA OF OVARY DETECTED ON ACCOUNT OF ACUTE APPENDICITIS
}

Avijeet Mukherjee, Naveen N, Manohar T. M, Gopal N.

1. Assistant Professor. Department of General Surgery, Adichunchanagiri Institute of Medical Sciences.

2. Assistant Professor. Department of General Surgery, Adichunchanagiri Institute of Medical Sciences.

3. Professor \& HOD. Department of General Surgery, Adichunchanagiri Institute of Medical Sciences.

4. Professor. Department of Obstetrics \& Gynaecology, Adichunchanagiri Institute of Medical Sciences.

\section{CORRESPONDING AUTHOR:}

Dr. Naveen. N, \#90, 14 $4^{\text {th }}$ Main, $14^{\text {th }}$ Cross,

$2^{\text {nd }}$ Stage, $2^{\text {nd }}$ Phase,

West of Chord Road,

Mahalakshmipuram, Bangalore - 560086.

E-mail: naveen_uno1@yahoo.co.in

ABSTRACT: Ovarian cancer accounts for $5 \%$ of all cancers among women and causes more deaths than any other female genital tract cancer. The majority (85-90\%) of ovarian cancers is epithelial in origin and arises typically in postmenopausal patients. An ovarian serous cystadenocarcinoma forms the malignant end of ovarian serous tumours. It is the most common malignant ovarian tumor and is derived from glandular epithelium, in which cystic accumulations of retained secretions are formed. Early diagnosis is often a matter of maintaining high clinical suspicion. Management consists initially of optimal surgical cytoreduction with or without chemotherapy.

Appendicitis is a polymicrobial infection of the appendix. Appendectomy for appendicitis is the most commonly performed emergency operation in the world. Elderly patients with appendicitis often pose a more difficult diagnostic problem because of the atypical presentation and expanded differential diagnosis such as a twisted ovarian tumor may erroneously be diagnosed as appendicitis in females.

In this paper, we present a case of a $58 \mathrm{yr}$ old female, who presented with features suggestive of acute appendicitis. On examination an abdomino-pelvic mass was revealed. On further investigation, a left ovarian serous cystadenocarcinoma in stage 1 was diagnosed. Laparotomy was done with removal of the left ovarian tumor, contralateral ovary, bilateral fallopian tubes and uterus.

KEYWORDS: Ovarian Cancer; Serous Cystadenocarcinoma; Malignant Epithelial Neoplasms; Appendicitis

INTRODUCTION: Ovarian cancer accounts for more deaths than all other gynecologic malignancies combined and are the fifth leading cause of cancer-related death ${ }^{1}$. Approximately $90 \%$ of malignant ovarian tumors in adults are of epithelial origin followed by sex cord stromal tumors $(6 \%)$ and germ-cell tumors (3\%). An ovarian serous cystadenocarcinoma forms the malignant end of ovarian serous tumors. They account for the largest proportion of malignant ovarian tumours ${ }^{2}$ and can account for over $50-80 \%$ of all malignant epithelial ovarian tumours ${ }^{3}$. The prevalence peaks around the $6^{\text {thto }} 7^{\text {th }}$ decades of life ${ }^{4}$. 
Early stage ovarian cancer patients present with nonspecific symptoms such as vague abdominal or pelvic pain, bloating, urinary symptoms, nausea, fatigue, and back pain may be noted ${ }^{5}$. Majority of cases are already at an advanced stage at presentation and diagnosis ${ }^{6}$. The four important symptoms include abdominal pain (frequency 30\%), abdominal swelling (frequency 16.5\%), gastrointestinal symptoms (frequency $8.5 \%$ ), and pelvic pain (frequency $5.4 \%)^{7}$.

CA-125 is a glycoprotein, produced by both benign and malignant ovarian tumors and is integral to management of ovarian cancer. Transvaginal sonography (TVS) is typically the most useful imaging test. The main advantage of computed tomography (CT) scanning is in advanced ovarian cancer, as it may detect disease in the liver, retroperitoneum, omentum, or elsewhere in the abdomen ${ }^{8}$.

Papillary serous cystadenocarcinomas are the most common form of malignant ovarian cancer making up 26 percent of ovarian tumours in women aged over 209 . As with most ovarian tumours, due to the lack of early signs of disease these tumours can be large when discovered and have often metastasized, often by spreading along the peritoneum ${ }^{10}$ and usually present with omental metastases which cause ascites.

Ovarian cancer staging is by the FIGO staging system and uses information obtained after surgery. AJCC staging system describes extent of the primary Tumor (T), absence or presence of metastasis to nearby lymph Nodes $(\mathrm{N})$, and absence or presence of distant Metastasis (M) ${ }^{11}$.

The treatment of ovarian cancer is based on the stage of the disease which is a reflection of the extent or spread of the cancer to other parts of the body and includes optimal debulking of disease followed by combination chemotherapy. The most common combinations at this time consist of a platinum agent and a taxane. The third modality is radiation treatment, which is used in only certain instances ${ }^{12}$.

The most important determinant for a favorable prognosis is diagnosis of ovarian carcinoma at an early stage. The prognosis of invasive ovarian cancer is poor, and relates to stage, tumor grade and residual disease after surgery. The prognosis of early-stage ovarian invasive cancers and borderline tumors of all stages is significantly better.

CASE REPORT: A $58 \mathrm{yr}$ old female patient presented to surgery OPD with pain abdomen, nausea, vomiting, and fever in that order of occurrence since the previous day evening. She had taken treatment from a local doctor but symptoms didn't subside. Pain was in lower abdomen on right (RT) side and was continuous. Associated with nausea, patient had 3 episodes of vomiting and fever of moderate degree. Patient had attained menopause 4 yrs back and was 13 yrs old at the time of menarche. She had 2 children. On examination, there was a mass of size $12 \times 16 \mathrm{cms}$ occupying the hypogastric, upto the umbilicus with well defined margins except the lower margin extending behind the pubis. Mass had smooth surface, was non tender and ballotable. Dull note was elicited over the mass. Bimanual pelvic examination confirmed it to be ovarian in origin. McBurney's point tenderness was present. On probing about the abdominal mass, patient revealed that the mass was noticed by her 9 months back and has gradually progressed from a smaller size to the current size and since it did not result in any problems in terms of pain, she ignored it. Patient had been having abdominal bloating, indigestion and backache which she attributed the symptoms to acid peptic disease and was on treatment for the same. 
Blood examination showed leucocytosis with neutrophilia and raised ESR. On ultrasound (USG) of abdomen and pelvis coupled with transvaginal ultrasonography (TVS), a solitary, multiloculated, large $(14.5 \times 20 \mathrm{cms})$ pelvic tumor with thick septations as well as papillary projections and echogenic (solid) areas arising from left (Lt) ovary was found. A non compressible appendix with wall thickening and periappendiceal fluid was also noted. CT findings were in lieu with USG and TVS and showed no evidence of intraabdominal metastases or ascites. Serum CA-125 level was 380 units $/ \mathrm{ml}$. Patient was prepared for surgery and a senior gynaecologist was called for. A vertical midline laparotomy incision was taken. Stomach, omentum, liver and undersurface of the diaphragm were evaluated and multiple cytologic pelvic washings were taken. Rt ovary and the uterus grossly appeared normal. The left ovarian tumor (Fig 1 \& 2) was removed in toto with intact capsule along with contralateral normal ovary, bilateral fallopian tubes, and uterus. Omentectomy with random peritoneal biopsies and retroperitoneal lymph node sampling was done. Appendectomy was done (Fig 3).

HPE of the specimen showed tumour being complex multi-loculated cyst with branching papillary fronds with exuberant solid areas in places and smooth capsule, well differentiated (Grade 1) with characteristic microscopic features including finger-like papillae with fibrovascular core, covered by multilayered cuboidal or columnar epithelium, hyperchromatic nuclei, Psammoma bodies and desmoplasia, so diagnosis of Papillary Serous Cystadenocarcinoma of the Lt ovary was done (Fig 4). Appendix specimen showed features suggestive of acute appendicitis (Fig 5). Specimens of Rt ovary, salpinges, uterus, omentum, retroperitoneal lymph nodes, peritoneal biopsies including cytologic intraperitoneal washings showed no evidence of involvement. Thus tumour was comprehensively staged as $1 \mathrm{~A}$ under AJCC and no further treatment was recommended to the patient in form of chemoradiotherapy. Patient is currently under surveillance with regular follow up for every three months since $1 \mathrm{yr}$ in terms of physical and pelvic examinations and CA-125 levels monitoring. Patient is doing fine and is been advised to continue follow up every 3 months for the subsequent year, then twice yearly for an additional 3 years, and then annually.

DISCUSSION: Ovarian cancer is the leading cause of death from gynecologic malignancies. It is typically portrayed as a 'silent killer' without appreciable signs or symptoms until advanced disease is obvious clinically. Actually, as in the above case, patients are often symptomatic for several months before the diagnosis, which they or their primary health providers ignore. The difficulty is in distinguishing these symptoms from those that occur normally in women and often these non-specific symptoms are ascribed to some other disease. Because early ovarian cancer produces few specific symptoms, most women present with advanced stage disease, for which the cost of treatment is high and the prognosis poor. Screening for ovarian cancer is not currently recommended for the general population but may be appropriate for those considered at high risk. Early stage disease carries an excellent prognosis ( $5 \mathrm{yr}$ survival $86 \%)^{13}$ and has the potential for cure. For the ovarian cancer to be detected in early stage the onus lies both on the patient and the treating physician, as in our case, where the sheer negligence of the patient in presenting early even when the mass was noticed which was "not causing her any harm" (as per the patient) and lack of clinical acumen on part of her treating doctor. A proper clinical history and a simple abdominal examination of the patient would have sufficed. So this case infers that an apt history taking and a sound clinical examination on part of the treating doctor with a high degree of suspicion for malignancy in the early stages of presentation is required. Only because of her appendix (acute appendicitis), the malignancy was detected at an initial stage and cure 
was possible. Good surgery is a blend of good judgment and sound surgical technique. So the surgeon has to make apt clinical decision concerning patient selection, choice of the right operation, and postoperative treatment while treating ovarian malignancy.

\section{REFERENCES:}

1. Jemal A, Siegel R, Ward E, et al: Cancer statistics, 2007. CA Cancer J Clin 57(1):43, 2007 [PMID: 17237035].

2. Hamm B, Forstner R, Beinder E et-al. MRI and CT of the Female Pelvis. Springer Verlag. (2007) ISBN: 3540222898.

3. Kawamoto S, Urban BA, Fishman EK. CT of epithelial ovarian tumors. Radiographics. 1999; 19 Spec No: S85-102.

4. Ros PR, Mortele KJ. CT and MRI of the abdomen and pelvis, a teaching file. Lippincott Williams \& Wilkins. (2006) ISBN: 0781772370.

5. Goff BA, Mandel LS, Melancon CH, et al: Frequency of symptoms of ovarian cancer in women presenting to primary care clinics. JAMA 291:2705, 2004 [PMID: 15187051].

6. Allen DG, Baak J, Belpomme D, et al. Advanced epithelial ovarian cancer: 1993 consensus statement. Ann Oncol 1993; 4(suppl 4): S83.

7. Aletti GD, Dowdy SC, Podratz KC, et al. Surgical treatment of diaphragm disease correlates with improved survival in optimally debulked advanced stage ovarian cancer. Gynecol Oncol 2006; 100:283.

8. American College of Obstetricians and Gynecologists: The role of the generalist obstetrician-gynecologist in the early detection of ovarian cancer. ACOG committee opinion no. 280, December, 2002. Obste Gynecol 100:1413, 2002.

9. Kosary, Carol L. (2007). 'Chapter 16: Cancers of the Ovary'. In Horner, M-J; Young, JL; Keel, GE et al. SEER Survival Monograph: Cancer Survival Among Adults: US SEER Program, 1988-2001, Patient and Tumor Characteristics. SEER Program. NIH Pub. No. 07-6215. Bethesda, MD: National Cancer Institute. pp. 133-144.

10. 'The Internet Pathology Laboratory for Medical Education'. The University of Utah Eccles Health Sciences Library. Retrieved 2009-06-27.

11. Goff, BA; Mandel, L; Muntz, HG; Melancon, CH (2000 Nov 15). 'Ovarian carcinoma diagnosis'. Cancer 89 (10): 2068-75.

12. Whitney CW: Gynecologic Oncology Group Surgical Procedures Manual. Gynecologic Oncology Group; available at https://gogmember.gog.org/manuals/pdf/surgman.pdf; accessed July 2005.

13. Heintz APM, Odicino F, Maisonneuve P, et al: Carcinoma of the ovary. In FIGO annual report on the results of treatment in gynaecological cancer. Int J Obstet Gynecol 95(Suppl 1):S161, 2006. 


\section{CASE REPORT}

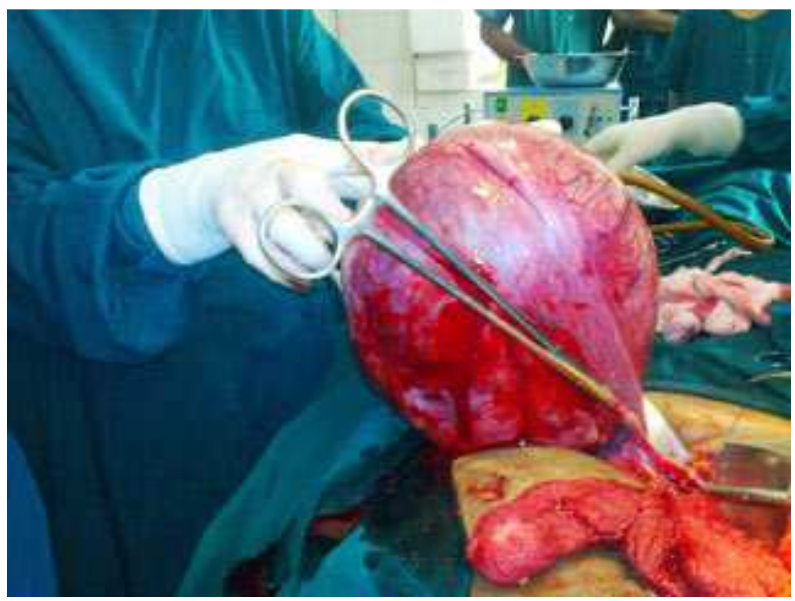

Fig 1 - Intraop picture of the large Lt ovarian serous cystadenocarcinoma

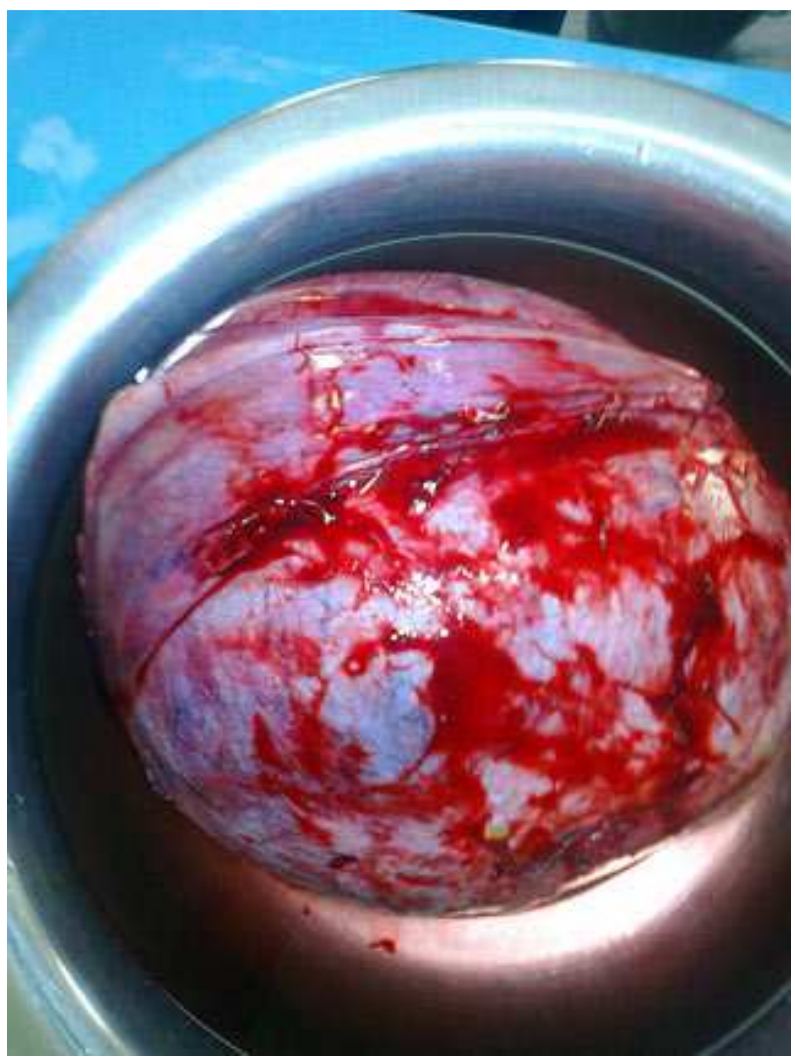

Fig 2 - Specimen measuring 15x21 cms removed with intact capsule 


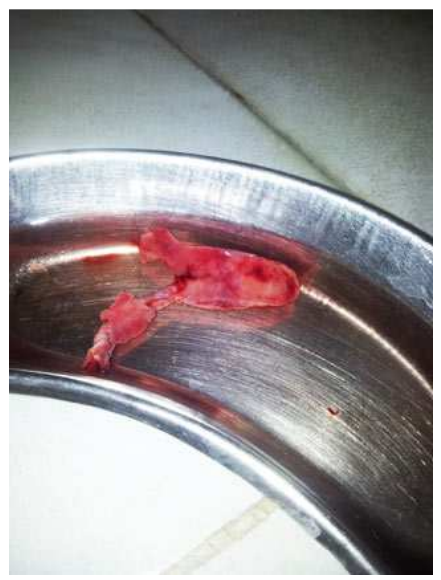

Fig 3 - Appendix Specimen

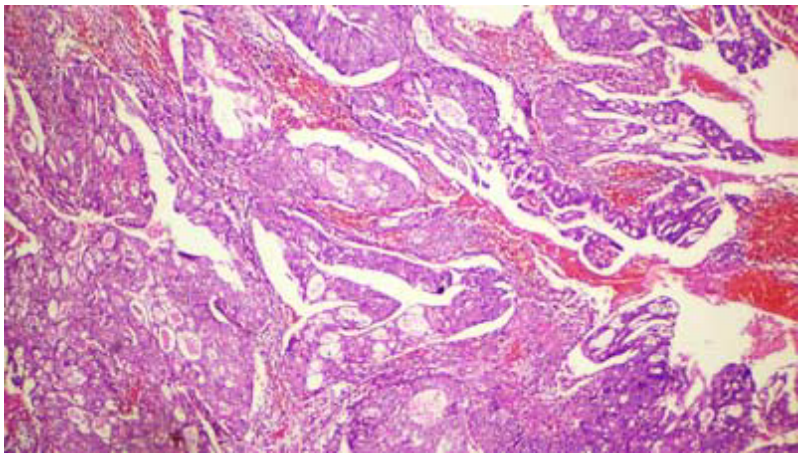

Fig 4 - Microscopic examination of the ovarian tumor shows numerous papillary processes lined by nonciliated cuboidal or columnar epithelium showing mild to moderate degree atypia, stratification and nests of tumours cells seen invading the underlying stroma. Also noted are cystic spaces filled with eosinophilic secretions, suggestive of low grade serous cystadenocarcinoma of ovary

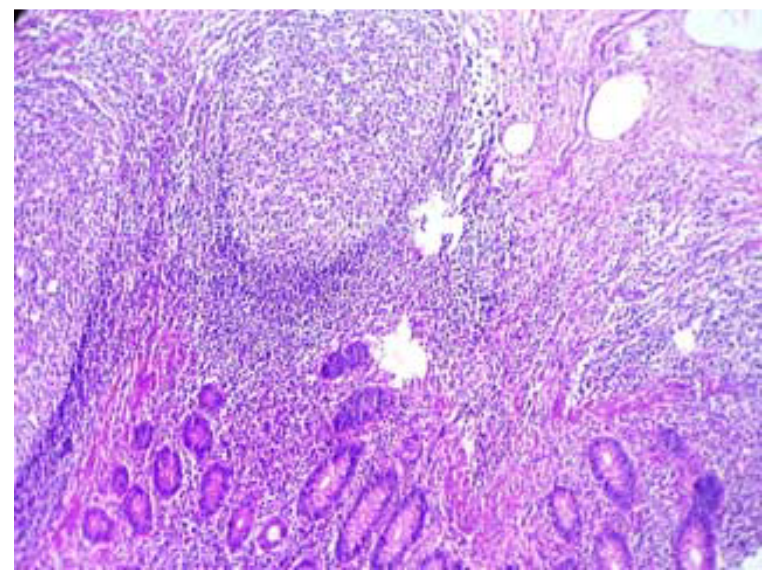

Fig 5 - Sections studies from appendix show reactive hyperplasia of follicles with few residual mucosal glands. Dense inflammatory cell infiltrate is seen in surrounding stroma predominantly composed of neutrophils and few lymphocytes, suggestive of acute appendicitis 